\title{
APPARATUS FOR THERMOMAGNETIC ANALYSIS
}

\author{
By Raymond L. Sanford
}

\section{ABSTRACT}

Thermomagnetic analysis consists in the study of magnetic effects occurring in a material during heating or cooling, with special reference to their interpretation in terms of structural changes or transformations. It is most conveniently carried out by means of a magnetometer of suitable type. The present paper describes thermomagnetic analysis apparatus set up at the Bureau of Standards and gives typical results obtained by its use.

\section{CONTENTS}

I. Introduction

II. General arrangement

III. The magnetometer

IV. Coils and furnace

V. Experimental procedure-

VI. Typical results

\section{INTRODUCTION}

The magnetic properties of ferromagnetic materials depend not only upon previous thermal history but also upon the existing temperature. Even such relatively small variations of temperature as may occur within the atmospheric range bring about easily measurable changes in magnetic properties which can not be neglected ${ }^{1}$ in work of high accuracy. Temperature changes of greater magnitude may produce alterations in the structure of the material accompanied by corresponding changes in magnetic properties. Observations of magnetic changes which occur during heating or cooling, when properly interpreted, therefore, are useful in the study of the constitution of ferromagnetic materials and the effect upon it of various treatments. The study of magnetic effects occurring during heating or cooling with special references to their interpretation in terms of structural changes or transformations is termed "thermomagnetic analysis."

Honda and his associates ${ }^{2}$ have found the methods of thermomagnetic analysis very valuable for the study of iron and steel and have even extended them to the study of paramagnetic materials.

${ }^{1}$ Sanford B. S. Sci. Paper No. 245 ; 1915. Spooner, Phys. Rev., 27, p. 183 ; 1326.

${ }^{2}$ Many papers in Sci. Rep. Tohoiru Univ., Sendai.; 1912, et seq. 
Other investigators ${ }^{3}$ have also employed thermomagnetic analysis to a certain extent, but thus far little use of this method of investigation has been made in the United States.

Much attention has been given in recent years to the relationships which exist between the magnetic properties of materials and their composition and structure. If sufficiently definite relationships are established and various experimental difficulties overcome, magnetic properties can be made the basis of nondestructive testing methods of great value. The term magnetic analysis has been adopted to distinguish magnetic testing of this type from that carried out solely for the purpose of determining the magnetic characteristics as such. Thermomagnetic analysis when properly developed should take its place as an important phase of the more general subject of magnetic analysis.

The apparatus described in the present paper was designed for the purpose of applying the methods of thermomagnetic analysis to the study of the structure of iron and steel as affected primarily by heat treatment. The problem was originally undertaken several year's ago by Dr. C. Nusbaum, who carried out a considerable amount of work in connection with the design and construction of the apparatus. Unfortunately, it was necessary to interrupt the work in faror of more urgent problems and, consequently, he was unable to carry it to completion. The work has recently been taken up again. The apparatus has been somewhat simplified and modified so as to meet the difficult experimental conditions under which it is necessary to work, but the main features follow, in general, the original design.

No especial originality is claimed for the method or equipment, but, in view of the peculiar difficulties encountered in attempting to use this type of apparatus in a location where there are so many magnetic disturbances as are found at the Bureau of Standards, it was thought that a description would be of some interest

\section{GENERAL ARRANGEMENT}

There are two ways in which thermomagnetic analysis may be carried out. The specimen may be held at a series of fixed temperatures and complete magnetization curves determined for each temperature, or a fixed value of magnetizing force may be applied and the changes in magnetization noted as the temperature is gradually raised or lowered. Each of these methods has its own particular advantages and disadvantages. For the present purpose the second method seems to be preferable because by its use it is possible to make continuous observations of the magnetic changes and to note not only their magnitude, but also the rate at which they progress

\footnotetext{
${ }^{3}$ Curie (Mme.), Bull. de la Soc. d'Encouragement, 3, p. 36 ; 1898. Morris, Phil. Mag., 44, p. 213; 1897. Terry, Phys. Ret., 28, p. 393; 1909. Smith, Phil. Trans. Roy. Soc. London, 215, p. 177; 1915. Dejean, Annal. de Phys., 9 ser., 18, p. 171; 1922.
} 


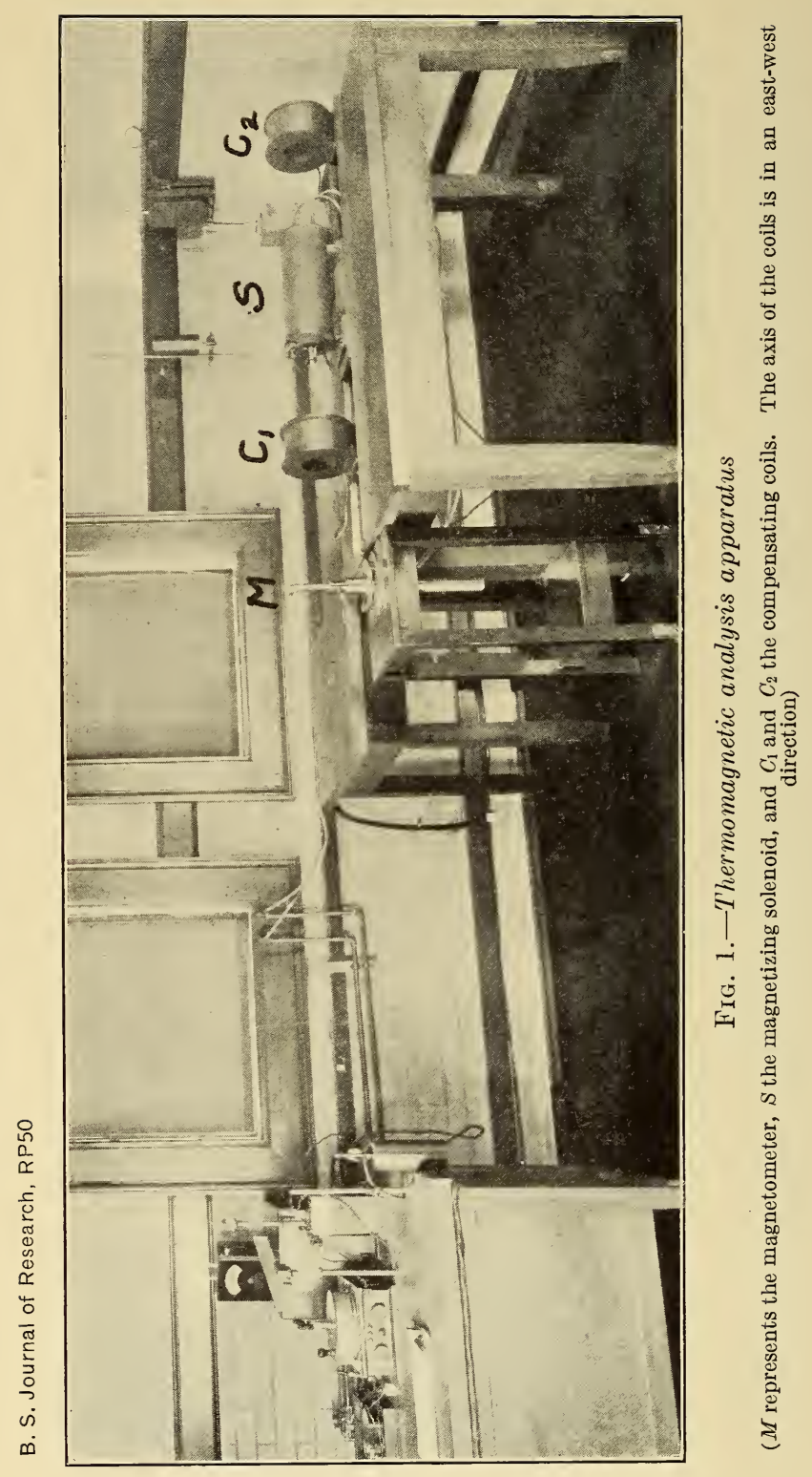


with change in temperature. It is also possible to study the efiect of rate of heating or cooling on the observed transformations.

For the continuous observation of relatively slow changes in magnetization such as occur during heating or cooling, the magnetometer is particularly suitable. With this method the magnetization is indicated by the deflection of a suspended magnetic needle placed in a definite position with respect to the test specimen. The direct action of the magnetizing solenoid is neutralized by suitable compensating coils. The sensitivity of a single needle magnetometer is determined not only by the strength of the needle, but also by the torsional strength of the suspension and the intensity of the earth's magnetic field. In the magnetic laboratory of the Bureau of Standards the field is continually varying both in direction and magnitude, due to outside disturbances from electrical machinery, elevators, and the like. Consequently, the use of a single needle is not practicable, and an astatic system must be used.

Figure 1 is a photograph of the apparatus which consists essentially of a magnetizing solenoid $S$, compensating coils $C_{1}$ and $C_{2}$, and the astatic magnetometer $M$. Within the solenoid $S$ is mounted an electric furnace. On a separate table are mounted rheostats for controlling the magnetizing current, an autotransformer for controlling the heating current, a potentiometer for temperature measurement, and lamps and scales for reading the potentiometer and magnetometer deflections. The coils are arranged with their axes in an east-west direction, and the magnetometer is east of the coils. The furnace is water jacketed and a platinum, platinum-rhodium thermocouple is used in the temperature measurements.

\section{THE MAGNETOMETER}

The construction of the magnetometer system is shown in Figure 2. The moving system is suspended by means of a quartz fiber from the threaded brass rod, $R$, which extends through the torsion head of the instrument. Vertical adjustment is provided by means of a nut on the head. A tangent screw is also provided for fine adjustment of the zero. The quartz fiber suspension $Q$ is approximately $8 \mathrm{~cm}$ long and $0.04 \mathrm{~mm}$ in diameter, and is attached to the rod with shellac. For the sake of convenience in making the necessary adjustments, the suspended system is in two parts connected by small hooks made of flat wire so as to prevent relative twisting of the two parts. The upper part of the system carries a plane mirror, $M$, $1 \mathrm{~cm}$ in diameter and a mica damping vane, $V$, as shown. The image of a lamp filament is focused on a ground glass scale by means of a lens of $2 \mathrm{~m}$ focal distance mounted in the case of the instrument. The mica vane hangs in a damping chamber and provides practically critical damping. 
In the lower system the magnetic needles are mounted in short brass tubes as shown. A brass rod $1 \mathrm{~mm}$ in diameter extends between

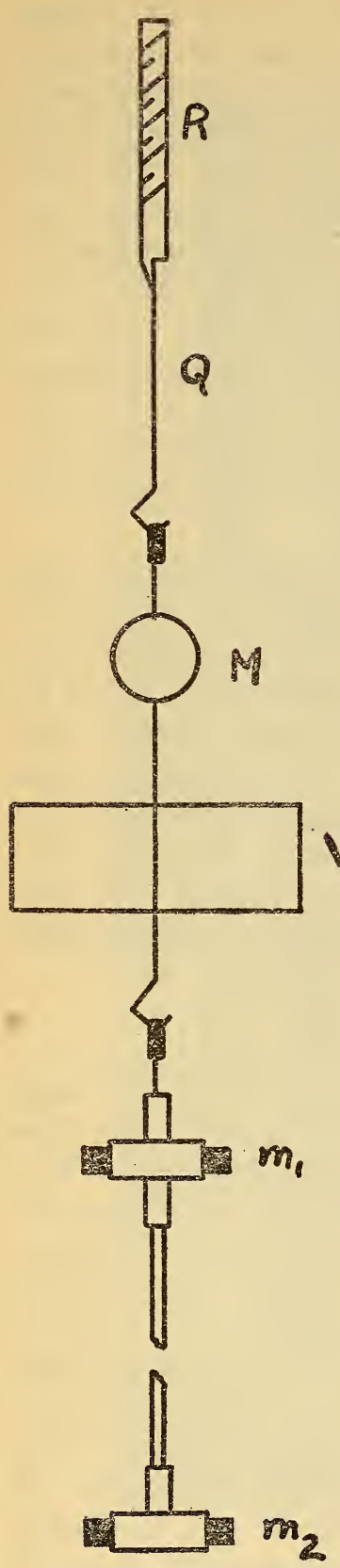

Fig. 2.-Magnetometer moving system the needles, and the distance can be varied by changing the length of the rod. The usual distance is approximately $25 \mathrm{~cm}$. As the distance rod is held by friction in tubular projections, the magnets can easily be oriented with respect to each other. This type of magnet system was chosen because, since there is a vertical gradient in the horizontal intensity of the magnetic field, it is desirable to be able to adjust the magnetic moments of the needles separately. The needles, $1 \mathrm{~cm}$ long and $3 \mathrm{~mm}$ in diameter, are made of special magnet steel containing about 15 per cent cobalt. This type of steel, when properly heat treated, has a coercive force from two to three times as great as that of the ordinary 5 per cent tungsten steel and for this reason is especially adapted for short, thick magnets. The magnetic moment of the needles has not been determined, but comparative tests indicate that the strength is about three times as great as could be obtained with the same weight of steel of the ordinary type. With an astatic system, the sensitivity depends directly upon the strength of the needles, so the stronger needles constitute a distinct advantage. The total weight of the suspended system is about $6 \mathrm{~g}$. The outer case of the magnetometer is made of sections of brass tubing which are removable so that the various parts of the suspended system are easily accessible.

In setting up and attempting to use the apparatus two difficulties were encountered which may be of interest to mention. As first set up the zero was found to drift badly. For several days the reading was brought back to zero each day by adjusting the torsion head, but the zero continually drifted in the same direction, sometimes as much as $5 \mathrm{~cm}$ in a day. Various parts of the apparatus were inves-
tigated in an attempt to locate the reason for this behavior, which tigated in an attempt to locate the reason for this behavior, which 
was finally traced to the supporting rod $R$. It was found that, apparently due to the relief of internal stresses, the rod was continually twisting. The trouble was eliminated by annealing the rod at $500^{\circ}$ C. The second difficulty was a tendency for the shellac to yield gradually, thus lowering the system and altering the sensitivity and compensation. This difficulty was obviated by heating the shellac to slightly above $100^{\circ} \mathrm{C}$. and holding it at this temperature overnight after application to the rod and suspension hook but previous to attaching the quartz fiber. One suspension mounted in this way has now been in use several months without signs of yielding.

On account of the variability of the field in which the magnetometer is located, its behavior depends largely upon the care with which the relative strengths and orientation of the needles are adjusted. Not only is the field variable, but also there is a vertical gradient in horizontal intensity. The gradient amounts to a variation in field strength of about 0.3 per cent per $\mathrm{cm}$ of vertical distance. It is necessary, therefore, in order to make the resultant torque zero, to adjust the strength of the needles so as to be inversely proportional to the respective strengths of the field in which they hang. This adjustment can be made only approximately, because the ratio of the field strengths at the two positions is constantly changing. The wandering of the zero due to this cause is a function of the variation in this ratio.

While it is essential to have the relative strengths of the needles properly adjusted, it is even more necessary to have their magnetic axes parallel. If there is an angle between the two axes, there is a resultant magnetic moment nearly at right angles to the needles, and any variation in the strength of the earth's field causes a wandering of the zero. In addition to this, if the magnetizing and compensating coils are adjusted to give no torque at the zero position, the compensation does not remain good if the system is deflected. The reason for this can be shown as follows:

Let

$T_{1}=$ torque of upper needle,

$T_{2}=$ torque of lower needle,

$\theta=$ angle between axis of coils and upper needle,

$\alpha=$ angle between axes of the needles,

then

$\theta+\alpha=$ angle between axis of coils and lower needle.

$$
\begin{aligned}
& T_{1}=k_{1} \sin \theta \\
& T_{2}=k_{2} \sin (\theta+\alpha)
\end{aligned}
$$

where $k_{1}$ and $k_{2}$ are constants. When the system is compensated

or

$$
T_{1}=T_{2}
$$

$$
k_{1} \sin \theta=k_{2} \sin (\theta+\alpha)
$$


This condition can hold good for more than one $\theta$ only when $\alpha=0$. In other words, in order for the compensation to remain good when the needles are deflected by the magnetic field of the specimen, the magnetic axes of the needles must be parallel to each other.

The adjustment of the magnetometer system is carried out as follows :

1. The magnet system is replaced by an equivalent nonmagnetic weight, and the torsion head is adjusted so as to bring the reading to zero. This adjustment is not subsequently altered.

2. The upper hook of the magnet system, which is held by friction, is then oriented so as to bring the needles approximately perpendicular to the earth's field when suspended, and the relative strengths of the needles are adjusted so that, when the system is suspended, the reading is zero, thus indicating that there is no resultant torque. This is most conveniently done by reducing the strength of the stronger needle by subjecting it to the influence of a powerful permanent magnet.

3. Next, the lower needle is removed and the hook so oriented as to bring the reading to zero when the system is suspended. In this position there is no torque on the suspension, as the needle is oriented in the direction of the earth's magnetic field.

4. The lower needle is then replaced and so oriented with aspect to the upper needle that when the system is again hung on the suspension the reading is zero, thus indicating that the torque due to each needle is completely balanced by that of the other. The system is then in proper adjustment.

In locations where there is considerable magnetic disturbance due to stray fields it is desirable to make the final adjustment at a time when the disturbances are a minimum, which is generally some time in the neighborhood of $3 \mathrm{a} . \mathrm{m}$. The adjustment of the system as at present constructed is rather tedious because there is no provision for twisting the various elements with respect to each other by known small amounts. It is possible that considerable improvement could be made in this respect, though it would be objectionable to increase the weight of the system.

As stated earlier, the behavior of the magnetometer is largely a function of the care with which the initial adjustments are made. On account of the nature of the variations in the magnetic field it is not possible to eliminate entirely the wandering of the zero. It has been possible, however, so to adjust the system that when the sensitivity is such as to give a maximum deflection of $20 \mathrm{~cm}$ the wandering is not over $1 \mathrm{~mm}$. The feasibility of improving this performance to any appreciable extent is rather doubtful. 


\section{COILS AND FURNACE}

The magnetizing solenoid consists of 16 layers of No. 12 double cotton covered copper wire wound on a brass form $36 \mathrm{~cm}$ in length. For convenience a tap was brought out from every fourth layer. The total number of turns is 2,364 , and the field at the center calculated on the basis of a coil of infinite length and the same number of turns per cm would be 83.1 times the current in amperes. In order to accommodate the furnace, however, the inside diameter has to be rather large, about $8 \mathrm{~cm}$, so that the actual value is nearly 5 per cent less. The magnetizing field in gilberts per $\mathrm{cm}$ at the center of the solenoid as calculated and checked experimentally is 79.2 times the current in amperes and for a distance of $12 \mathrm{~cm}$ at the center does not vary by more than 2 per cent.

The compensating coils are round on brass forms $10 \mathrm{~cm}$ long of approximately the same inside diameter as the main magnetizing coil and have 22 layers of No. 12 double cotton covered wire. It was at first intended to locate one compensating coil on the opposite side of the magnetometer from the magnetizing coil, but as finally set up it was found more convenient to have it on the same side. Consequently, since this coil must be quite near the magnetometer, only the outside layer of the winding is actually used.

The three coils are mounted on bases which slide on bronze tracks permanently secured to the table. The coils were carefully oriented so as to be coaxial, as any angle between the axis of the magnetizing and compensating coil would give a component of field in the direction of the magnetometer needles. If this condition exists, the sensitivity varies with the current in the coils (the Erhardt effect). ${ }^{4}$

The furnace consists of a bifilar winding of platinum strip wound on an alundum tube approximately $2 \mathrm{~cm}$ in diameter. This winding is noninductive to such a degree that with 5 amperes d. c. flowing there is no appreciable effect on the magnetometer. To prevent short circuiting, the winding is held in place on the tube by means of alundum cement.

Alternating current for heating is provided by an autotransformer with which the supply voltage can be regulated in steps of 0.1 volt. This arrangement gives exceptionally good control of rate heating or cooling. The temperature in the furnace is uniform to within $3^{\circ} \mathrm{C}$. over a length of approximately $12 \mathrm{~cm}$ at the middle.

Surrounding the furnace is a brass water jacket to which water is brought by means of brass pipe and rubber tubing. The cooling is very effective, no rise in temperature being observed in the magnetizing winding even with the furnace operating at $1,000^{\circ} \mathrm{C}$.

${ }^{4}$ Gray and Ross, An Improved Form of Magnetometer, Proc. Roy. Soc. Edinburgh, 29, p. $182 ; 1908-9$. 
The specimens are supported within the furnace by means of a glazed porcelain tube $1 \mathrm{~cm}$ in inside diameter and $50 \mathrm{~cm}$ long. Arrangements can easily be made for heating in a vacuum or in a gaseous atmosphere.

Temperatures are measured by means of a Pt, Pt-10 per cent Rh thermocouple, and a Leeds \& Northrup type $\mathbf{K}$ potentiometer. The cold junctions are maintained at $0^{\circ} \mathrm{C}$. by means of an ice box in the usual way.

\section{EXPERIMENTAL PROCEDURE}

Observations are ordinarily made in the following way: First, the magnetizing coil is set at the required distance from the magnetometer, and, with no specimen in the coil, the compensating coils are adjusted so as to exactly neutralize the effect of the magnetizing coil. The final adjustment is made by moving the coil $\mathrm{C}_{2}$. The sample and thermocouple are then inserted and the reading of the magnetometer noted when the magnetizing current has been set to the proper value. After starting the circulation of the cooling water the heating current is turned on and adjusted to such a value as to give the desired heating rate. The potentiometer is then set for the first temperature at which an observation is to be taken and the reading of the magnetometer is noted when the given temperature is reached. The potentiometer is then set for the next temperature and só on.

The results obtained depend somewhat upon the form of specimen and in particular upon the ratio of length to diameter. For a cylindrical specimen in a uniform magnetic field the effective magnetizing force is less than the impressed force.

where

$$
H=H^{\prime}-N I
$$

$H=$ the effective magnetizing force,

$H^{\prime}=$ the impressed magnetizing force,

$N=$ the demagnetizing factor, and

$I=$ the intensity of magnetization.

The factor $N$ varies with the dimensional ratio. Since the intensity of magnetization depends upon the susceptibility of the material and the susceptibility varies with temperature, it is obvious that, as the test proceeds, the effective magnetizing force also varies. For specimens having a small ratio of length to diameter there is a tendency for the effect of a change in susceptibility to be neutralized by the resulting change in the value of the effective magnetizing force. Under these conditions the effect of structural changes may appear to be so small as to go unnoticed. 
In view of the fact that other apparatus in use in the laboratory for testing small samples is adapted for specimens $6 \mathrm{~mm}$ in diameter, this was chosen as the standard diameter for this test. In order to have as uniform temperature conditions as possible it is advisable to use the shortest specimen which will give satisfactory results. By experiment it was found that the specimens should be at least $8 \mathrm{~cm}$ long, and preferably $10 \mathrm{~cm}$. Accordingly, the specimens are usually $6 \mathrm{~mm}$ in diameter and $10 \mathrm{~cm}$ long with a small hole drilled axially in one end for insertion of the thermocouple.

It is desirable to work with the distance from the specimen to the magnetometer as great as possible, for under this condition the compensation is most easily adjusted and maintained.

It is generally not necessary to know the exact value of magnetization of the specimen, as the significant data are the temperatures at which definite changes in magnetization occur. These are shown by the form of the curve obtained by plotting magnetization, represented by magnetometer deflection, against the corresponding temperature. However, when calibration is desired, it can most easily be accomplished by means of a specimen of known magnetic properties. This procedure has been found preferable to the use of a standard coil on account of the short distance between the magnetometer needles and the finite length of the specimen.

\section{TYPICAL RESULTS}

In Figures 3 to 7, inclusive, are shown typical results obtained with the apparatus. Figure) 3 represents the magnetic behavior of pure iron when heated above the transformation temperature. For the magnetizing force used, 200 gilberts per $\mathrm{cm}$, there is no appreciable drop in magnetization until a temperature of approximately $725^{\circ} \mathrm{C}$. is reached. From then on the drop is rapid to about $775^{\circ} \mathrm{C}$. The approach to the zero value is more gradual and it is usually very difficult to determine the exact temperature at which magnetization completely disappears. This is characteristic of all ferromagnetic materials.

Nickel, as indicated in Figure 4, shows somewhat different characteristics in that the magnetization begins to drop immediately upon the start of heating. The magnetic transformation temperature of nickel is much lower than that of iron. Magnetization is practically completely lost for the sample of Figure 4 at a temperature of approximately $330^{\circ}$ C. The applied magnetizing force was 240 gilberts per $\mathrm{cm}$.

When a material is made up of two or more magnetically distinct constituents having different magnetic transformation temperatures, their presence may be detected by means of the resulting breaks in 


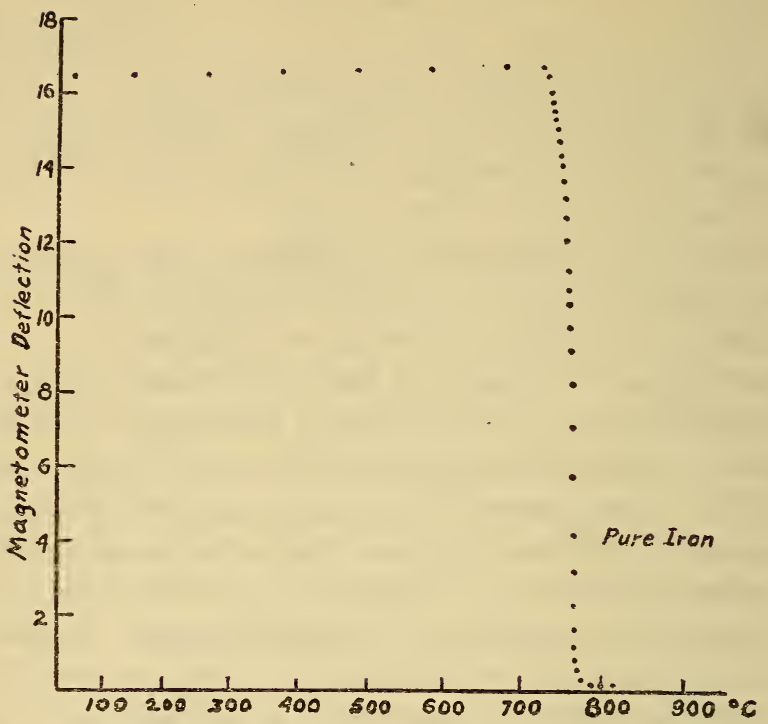

E'IG. 3.-Heating curve for puré iron Applied magnetizing force, 200 gilborts per $\mathrm{cm}$

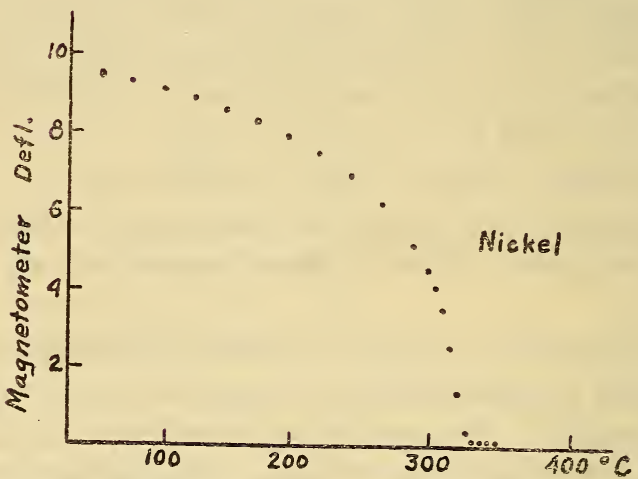

HIG. 4.-Heating curve for comnercially pure nickel

Applied magnetizing force, 240 gilberts per $\mathrm{cm}$ 


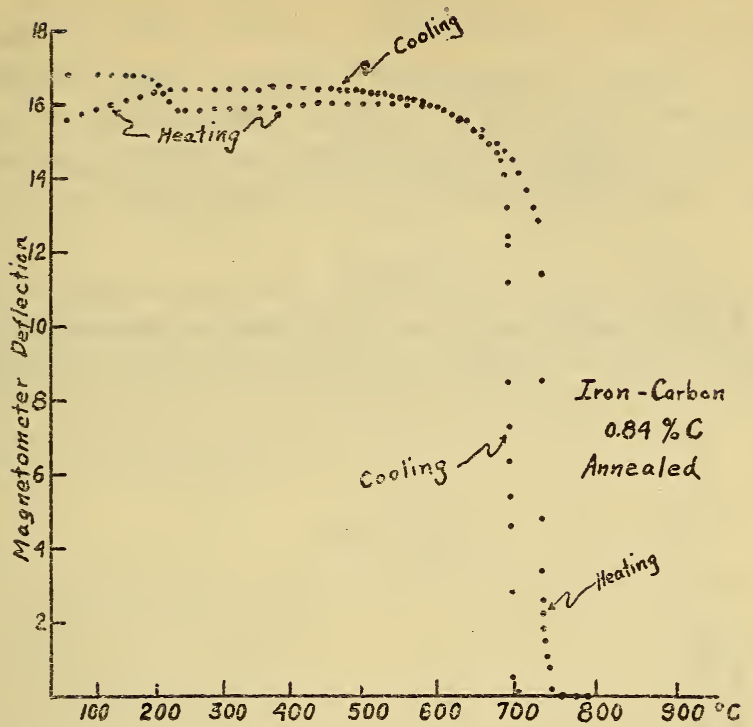

FIG. 5.-Heating and cooling curves for annealed troncarbon alloy of eutectoid composition

Applied magnetizing forces, 200 gilberts per cin

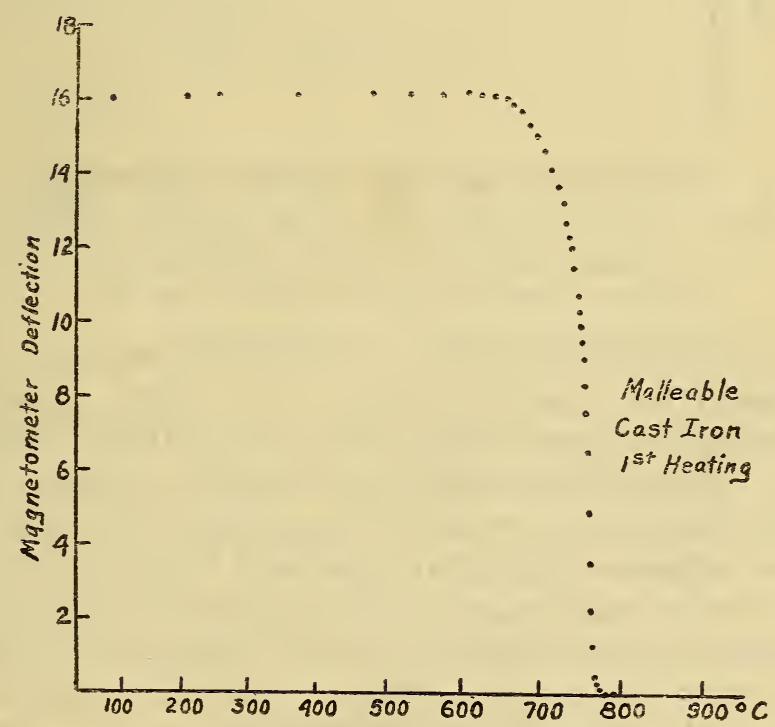

FIG. 6.-Heating curve for malleable cast iron Applied magnetizing force, 160 gilberts per $\mathrm{cm}$ 
the magnetization-temperature curve. One such instance is shown in Figure 5. The break in the region of $215^{\circ} \mathrm{C}$. is due to the transformation of the iron carbide, $\mathrm{Fe}_{3} \mathrm{C}$, present in the annealed carbon alloy. Iron carbide is magnetically distinct from iron, has a much lower saturation value, and a magnetic transformation temperature of about $215^{\circ} \mathrm{C}$. Figure 5 also illustrates the lag generally found between the loss of magnetization on heating and the recovery on cooling.

Figures 6 and 7 represent runs on the same sample of malleable cast iron. In malleable cast iron the carbon is normally in the form of graphite and not combined with iron to form the carbide. In

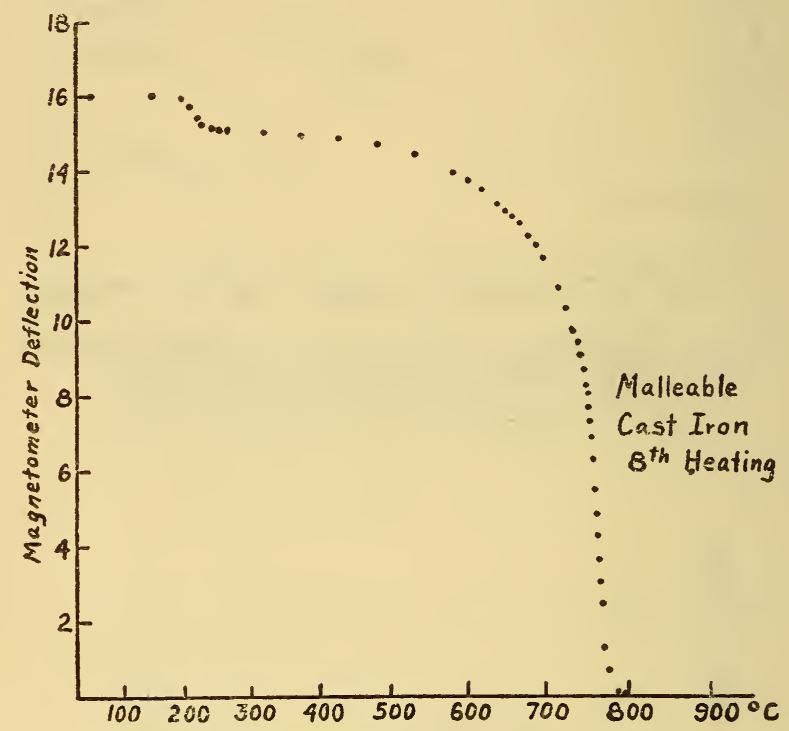

FIG. 7.-Heating curve for malleable cast iron previously heated seven times to $800^{\circ} \mathrm{C}$.

Applied magnetizing force, 160 gilberts per $\mathrm{cm}$

Figure 6 there is no trace of the carbide transformation at $215^{\circ} \mathrm{C}$., but in Figure 7 the transformation is very evident. The specimen had been heated to approximately $800^{\circ} \mathrm{C}$. seven times before the run of Figure 7. Some of the carbon has evidently combined with iron to form the carbide as the result of these repeated heatings.

There are influences other than structural transformations which may lead to variations in magnetic characteristics, so that the data of thermomagnetic analysis should always be interpreted with caution. It appears, however, that this hitherto somewhat neglected method should be capable of yielding significant and valuable results.

WAshington, October 1, 1928. 